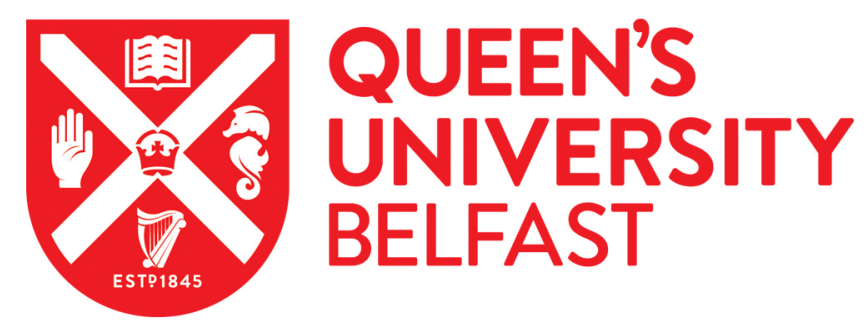

\title{
1ES 1927+654: An AGN Caught Changing Look on a Timescale of Months
}

Trakhtenbrot, B., Arcavi, I., MacLeod, C. L., Ricci, C., Kara, E., Graham, M. L., Stern, D., Harrison, F. A., Burke, J., Hiramatsu, D., Hosseinzadeh, G., Howell, D. A., Smartt, S. J., Rest, A., Prieto, J. L., Shappee, B. J., Holoien, T. W. S., Bersier, D., Filippenko, A. V., ... Loewenstein, M. (2019). 1ES 1927+654: An AGN Caught Changing Look on a Timescale of Months. Astrophysical Journal, 883(1), [94]. https://doi.org/10.3847/1538-4357/ab39e4

Published in:

Astrophysical Journal

Document Version:

Publisher's PDF, also known as Version of record

Queen's University Belfast - Research Portal:

Link to publication record in Queen's University Belfast Research Portal

Publisher rights

(c) 2019. The American Astronomical Society. All rights reserved. This work is made available online in accordance with the publisher's policies. Please refer to any applicable terms of use of the publisher.

\section{General rights}

Copyright for the publications made accessible via the Queen's University Belfast Research Portal is retained by the author(s) and / or other copyright owners and it is a condition of accessing these publications that users recognise and abide by the legal requirements associated with these rights.

Take down policy

The Research Portal is Queen's institutional repository that provides access to Queen's research output. Every effort has been made to ensure that content in the Research Portal does not infringe any person's rights, or applicable UK laws. If you discover content in the Research Portal that you believe breaches copyright or violates any law, please contact openaccess@qub.ac.uk. 


\title{
1ES 1927+654: An AGN Caught Changing Look on a Timescale of Months
}

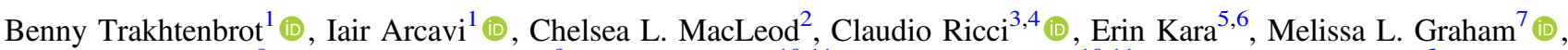 \\ Daniel Stern $^{8}$ (D), Fiona A. Harrison ${ }^{9}$, Jamison Burke ${ }^{10,11}$, Daichi Hiramatsu ${ }^{10,11}$, Griffin Hosseinzadeh ${ }^{2}$ (D), \\ D. Andrew Howell ${ }^{10,11}$ (D), Stephen J. Smartt ${ }^{12}$ (D), Armin Rest ${ }^{13,14}$, Jose L. Prieto ${ }^{3,15}$ (D), Benjamin J. Shappee ${ }^{16}$ (D), \\ Thomas W.-S. Holoien ${ }^{17}$ (D), David Bersier ${ }^{18}$ (D), Alexei V. Filippenko ${ }^{19,20}$ (D) , Thomas G. Brink ${ }^{19}$, WeiKang Zheng ${ }^{19}$ (DD, \\ Ruancun $\mathrm{Li}^{4}$, Ronald A. Remillard ${ }^{21}$, and Michael Loewenstein ${ }^{6,22}$ \\ ${ }^{1}$ School of Physics and Astronomy, Tel Aviv University, Tel Aviv 69978, Israel; benny@astro.tau.ac.il \\ ${ }^{2}$ Center for Astrophysics, Harvard \& Smithsonian, 60 Garden Street, Cambridge, MA 02138-1516, USA \\ ${ }^{3}$ Núcleo de Astronomía de la Facultad de Ingeniería, Universidad Diego Portales, Av. Ejército Libertador 441, Santiago, Chile \\ ${ }^{4}$ Kavli Institute for Astronomy and Astrophysics, Peking University, Beijing 100871, People's Republic of China \\ ${ }^{5}$ Joint Space-Science Institute, University of Maryland, College Park, MD 20742, USA \\ ${ }^{6}$ Astrophysics Science Division, NASA Goddard Space Flight Center, 8800 Greenbelt Road, Greenbelt, MD 20771, USA \\ ${ }^{2}$ Department of Astronomy, University of Washington, Box 351580, U.W., Seattle, WA 98195-1580, USA \\ 8 Jet Propulsion Laboratory, California Institute of Technology, 4800 Oak Grove Drive, MS 169-224, Pasadena, CA 91109, USA \\ ${ }^{9}$ Cahill Center for Astronomy and Astrophysics, California Institute of Technology, 1200 E. California Blvd, Pasadena, CA 91125, USA \\ ${ }^{10}$ Las Cumbres Observatory, 6740 Cortona Drive, Suite 102, Goleta, CA 93117-5575, USA \\ ${ }^{11}$ Department of Physics, University of California, Santa Barbara, CA 93106-9530, USA \\ ${ }^{12}$ Astrophysics Research Centre, School of Mathematics and Physics, Queens University Belfast, Belfast BT7 1NN, UK \\ ${ }^{13}$ Space Telescope Science Institute, 3700 San Martin Drive, Baltimore, MD 21218, USA \\ ${ }^{14}$ Department of Physics \& Astronomy, Johns Hopkins University, 3400 North Charles Street, Baltimore, MD 21218, USA \\ ${ }^{15}$ Millennium Institute of Astrophysics, Santiago, Chile \\ ${ }^{16}$ Institute for Astronomy, University of Hawai'i, 2680 Woodlawn Drive, Honolulu, HI 96822, USA \\ ${ }_{17}$ The Observatories of the Carnegie Institution for Science, 813 Santa Barbara Street, Pasadena, CA 91101, USA \\ ${ }^{18}$ Astrophysics Research Institute, Liverpool John Moores University, 146 Brownlow Hill, Liverpool L3 5RF, UK \\ ${ }^{19}$ Department of Astronomy, University of California, Berkeley, CA 94720-3411, USA \\ ${ }^{20}$ Miller Senior Fellow, Miller Institute for Basic Research in Science, University of California, Berkeley, CA 94720, USA \\ ${ }^{21}$ MIT Kavli Institute for Astrophysics and Space Research, 70 Vassar Street, Cambridge, MA 02139, USA \\ ${ }^{22}$ Department of Astronomy, University of Maryland, College Park, MD 20742, USA \\ Received 2019 March 25; revised 2019 June 25; accepted 2019 July 13; published 2019 September 24
}

\begin{abstract}
We study the sudden optical and ultraviolet (UV) brightening of 1ES 1927+654, which until now was known as a narrow-line active galactic nucleus (AGN). 1ES 1927+654 was part of the small and peculiar class of "true Type2" AGNs that lack broad emission lines and line-of-sight obscuration. Our high-cadence spectroscopic monitoring captures the appearance of a blue, featureless continuum, followed several weeks later by the appearance of broad Balmer emission lines. This timescale is generally consistent with the expected light travel time between the central engine and the broadline emission region in (persistent) broadline AGN. Hubble Space Telescope spectroscopy reveals no evidence for broad UV emission lines (e.g., C IV $\lambda 1549, \mathrm{C}$ III] $\lambda 1909, \mathrm{Mg}$ II $\lambda 2798$ ), probably owing to dust in the broadline emission region. To the best of our knowledge, this is the first case where the lag between the change in continuum and in broadline emission of a "changing look" AGN has been temporally resolved. The nature and timescales of the photometric and spectral evolution disfavor both a change in line-of-sight obscuration and a change of the overall rate of gas inflow as driving the drastic spectral transformations seen in this AGN. Although the peak luminosity and timescales are consistent with those of tidal disruption events seen in inactive galaxies, the spectral properties are not. The X-ray emission displays a markedly different behavior, with frequent flares on timescales of hours to days, and will be presented in a companion publication.
\end{abstract}

Key words: galaxies: active - galaxies: individual (1ES 1927+654) - galaxies: nuclei - quasars: emission lines quasars: general

Supporting material: data behind figures

\section{Introduction}

Large-scale time-domain surveys have allowed the recent identification of new types of extreme variability among both dormant and active supermassive black holes (SMBHs). Among these, so-called "changing look active galactic nuclei" (CL-AGNs, hereafter) are characterized by a switch between spectral states that are dominated by an AGN-like, power-law optical/ultraviolet (UV) continuum and/or strong broad emission lines ( $>1000 \mathrm{~km} \mathrm{~s}^{-1}$; i.e., Type-1 AGN), and those dominated by stellar continuum emission (from the host galaxy), and only narrow forbidden and permitted transitions from low- and high-ionization species (i.e., Type-2 AGN). Many recent studies have identified a growing number of such cases (e.g., Denney et al. 2014; Shappee et al. 2014; LaMassa et al. 2015; MacLeod et al. 2016; McElroy et al. 2016; Runnoe et al. 2016; Ross et al. 2018; Stern et al. 2018; Wang et al. 2018; Yang et al. 2018, and references therein), as well as cases of drastic changes to line-of-sight obscuration, identified in the X-rays (e.g., Matt et al. 2003; Piconcelli et al. 2007; Ricci et al. 2016).

The drastic changes seen in CL-AGNs challenge the simplest form of the AGN unification framework (e.g., Antonucci 1993; Urry \& Padovani 1995), where the two main spectral types are 
explained by orientation, and where Type-2 AGN are the dustobscured counterparts to broadline Type-1 AGN. A transient event in which a dusty cloud is going into (or out of) the line of sight is a rather unlikely explanation for CL-AGN, as the cloud would have to cover a significant fraction of the broadline emission region (i.e., BLR), with sizes $\gg 10^{16} \mathrm{~cm}$, to account for the Type- 2 appearance. More likely explanations are that CL-AGNs are driven by dramatic changes to the accretion rate onto the SMBH, or indeed the reformation or truncation of a radiatively efficient accretion flow; and/or by a sudden change to the amount of dense circumnuclear gas, which would give rise to broad emission lines (that is, BLR gas). The timescales relevant for the CL-AGNs reported up until now, of order several years, are far shorter than what is expected for global accretion rate changes in optically thick, geometrically thin accretion disks (see discussion in, e.g., Lawrence 2018; Stern et al. 2018, as well as the historical commentary given by Antonucci 2018). However, the sparse spectroscopic sampling of these CL-AGNs - with "before" and "after" spectra usually taken years apart-has so far prohibited a direct and clear-cut test of these scenarios.

1ES $1927+654$ is a known redshift $z=0.019422$ AGN, based on its X-ray emission, identified with the Einstein and ROSAT satellites. The strong, narrow forbidden lines of highionization species ([O III] $\lambda 5007$, [N II] $\lambda 6584$ ), observed in optical spectra taken in 2001 June, further classify 1ES 1927 +654 as a Type-2 AGN (e.g., Boller et al. 2003; Tran et al. 2011). Several studies of 1 ES $1927+654$ have suggested that it challenges the AGN unification framework, by pointing out the very small amount of obscuring material along the line of sight (i.e., a hydrogen column density of $\log \left[N_{\mathrm{H}} / \mathrm{cm}^{-2}\right] \lesssim 21.1$; see Gallo et al. 2013) and the lack of broad lines seen in polarized light (Tran et al. 2011). Thus, 1ES 1927+654 was proposed to be a prime example for the rare class of "true" or "naked" Type-2 AGNs, intrinsically lacking the denser gas that gives rise to the BLR, and/or the photoionizing continuum radiation that drives the broadline emission (see the very recent study by Bianchi et al. 2019).

Here we report a dramatic optical and UV brightening of 1ES $1927+654$, accompanied by the appearance of prominent broad emission lines with a time delay consistent with the expected size of a BLR.

\section{Observations}

\subsection{Photometry}

An increase in optical flux from 1ES 1927+654 was discovered on 2018 March 3 (UT dates are used throughout; Nicholls et al. 2018; Stanek 2018) and announced by the AllSky Automated Survey for Supernovae (ASAS-SN; Shappee et al. 2014), with a reported $V$-band host-subtracted magnitude of 15.3. The transient event was reported as ASASSN-18el and given the name AT 2018zf by the transient name server. ${ }^{23}$ Prediscovery detections from 2017 December 23 were recovered by the Asteroid Terrestrial-impact Last Alert System (ATLAS; Tonry et al. 2018) and designated as ATLAS18mgv. In what follows, we adopt this earlier ATLAS pre-discovery measurement as the detection date.

ASAS-SN images were processed by the fully automatic ASAS-SN pipeline (Shappee et al. 2014) using the ISIS image

\footnotetext{
${ }^{23}$ http://wis-tns.weizmann.ac.il/object/2018zf
}

subtraction package (Alard \& Lupton 1998; Alard 2000). We performed aperture photometry on the subtracted, stacked images in order to increase the signal-to-noise ratio of the detections, and calibrated the results using the AAVSO Photometric All-sky Survey (Henden et al. 2016).

Forced photometry was run on all reference-subtracted ATLAS images to produce magnitudes and $3 \sigma$ upper limits, using automated point-spread-function fitting, as documented by Tonry et al. (2018; all ATLAS magnitudes are in the AB system, Oke \& Gunn 1983). In a large number of images, there were flux artifacts at the transient position, due to the bright host and imperfect subtraction. These clearly spurious measurements were excised from the ATLAS light curve.

All optical magnitudes, as well as UV magnitudes and optical spectra (see below), were corrected for Milky Way extinction using $E(B-V)=0.077 \mathrm{mag}$ (Schlafly \& Finkbeiner 2011), ${ }^{24}$ the Cardelli et al. (1989) extinction law, and $R_{V}=3.1$.

Following the sudden optical flux increase, we initiated a near-UV (NUV) and X-ray monitoring campaign using the Neil Gehrels Swift Observatory (PI: I. Arcavi). UV/Optical Telescope (UVOT) photometry was extracted with standard HEASARC functions using a $5^{\prime \prime}$-radius aperture for the object and for the sky region. The host flux in the NUV $U V W 1$ and $U V M 2$ bands was determined from XMM-Newton/Optical Monitor (OM) data obtained in 2011 May 20 (Gallo et al. (2013), AB magnitudes of 18.14 and 18.65, respectively, using the same aperture), and subtracted from our new UVOT measurements. The X-ray analysis is discussed in detail in a companion paper (C. Ricci et al. 2019, in preparation). The final light curves are presented in Figures 1 and 2.

\subsection{Spectroscopy}

We obtained optical spectra of 1ES 1927+654 with the Ohio State Multi-Object Spectrograph (OSMOS) mounted on the Hiltner $2.4 \mathrm{~m}$ Telescope at the MDM Observatory (Martini et al. 2011); the Spectrograph for the Rapid Acquisition of Transients (SPRAT) instrument mounted on the $2 \mathrm{~m}$ Liverpool Telescope at the Roque de los Muchachos Observatory (Piascik et al. 2014); the Kast spectrograph mounted on the $3 \mathrm{~m}$ Shane Telescope at the Lick Observatory (Miller \& Stone 1993); the FLOYDS spectrograph mounted on the $2 \mathrm{~m}$ Faulkes Telescope North at Haleakala, Hawaii (part of the Las Cumbres Observatory network; Brown et al. 2013); the Dual Imaging Spectrograph mounted on the Apache Point Observatory $3.5 \mathrm{~m}$ telescope; the Low Resolution Imaging Spectrograph (LRIS) mounted on the Keck I telescope at Maunakea (Oke et al. 1995); and the Double Beam Spectrograph (DBSP) mounted on the Palomar Hale $5 \mathrm{~m}$ telescope (Oke \& Gunn 1982). All optical spectra were reduced following standard procedures, and scaled to match the ATLAS orange-band measurements, linearly interpolating between the nearest relevant ATLAS visits. Whenever possible, the spectra were taken with slits rotated to the parallactic angle, to minimize chromatic losses and spectral distortions (Filippenko 1982). The sequence of optical spectra is shown in Figure 3.

We obtained far-UV (FUV) and NUV spectra of 1ES 1927 +654 using the Cosmic Origins Spectrograph (COS) and Space

\footnotetext{
$\overline{24}$ Retrieved via the NASA/IPAC Extragalactic Database (NED): http://ned. ipac.caltech.edu/.
} 


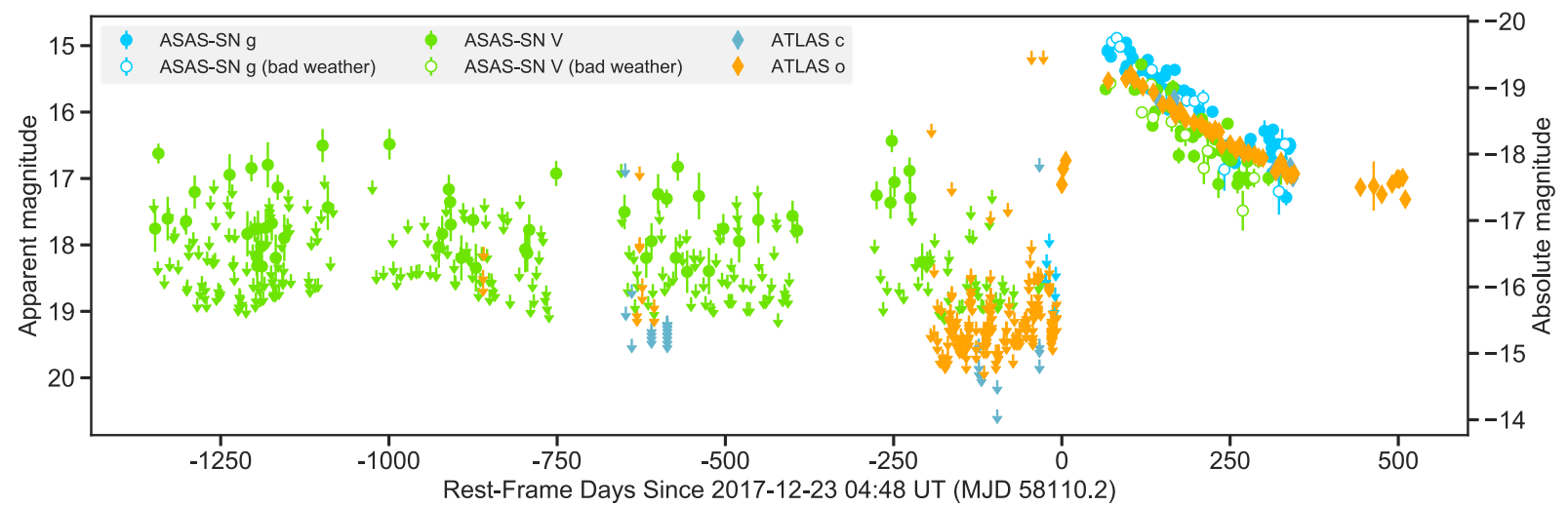

Figure 1. Long-term optical light curve of 1ES 1927+654 showing no activity at the level of the most recent flare (2017 December 23) in the last $\sim 4$ yr. Arrows denote $3 \sigma$ nondetection upper limits. All detections are binned nightly, and we separate ASAS-SN detections where clouds are seen in visual inspection of the images (marked by empty symbols). Error bars denote $1 \sigma$ uncertainties and are sometimes smaller than the symbols. All magnitudes are corrected for Milky Way extinction.

(The data used to create this figure are available.)

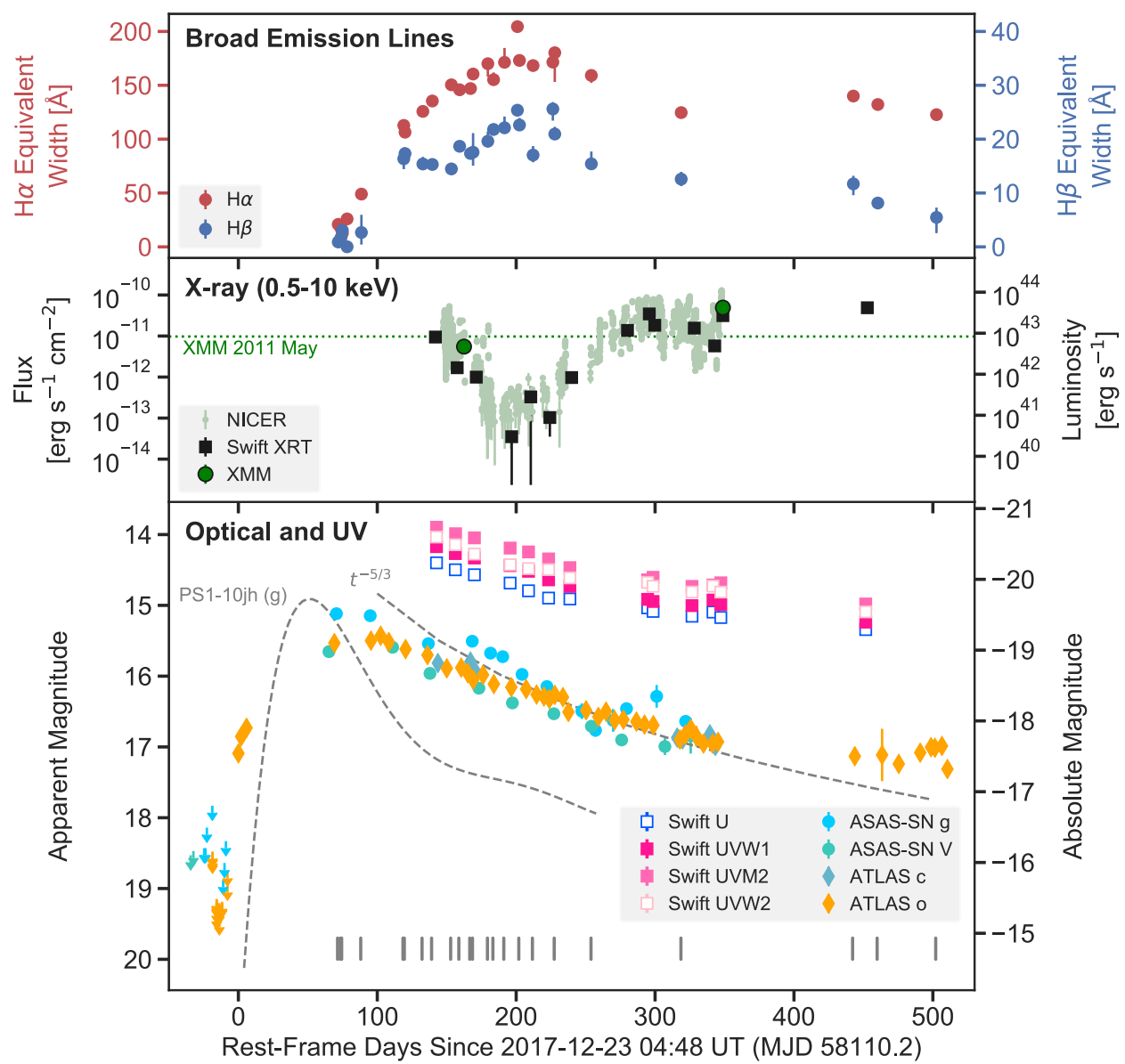

Figure 2. Light curve and equivalent-width evolution of 1ES 1927+654 during the flare. Bottom: optical and UV light curves, obtained with ATLAS, ASAS-SN, and Swift/UVOT. Arrows denote $3 \sigma$ nondetection upper limits. Empty symbols denote non-host-subtracted data. The ATLAS data are binned nightly up to peak, and then binned every two nights. The ASAS-SN data are binned weekly. Error bars denote $1 \sigma$ uncertainties. All magnitudes are corrected for Milky Way extinction. Vertical lines near the bottom of the panel denote epochs of optical spectra. The dashed gray lines trace a simple $\propto t^{-5 / 3}$ power law and the $g$-band light curve of the TDE PS110jh (matched in absolute magnitude; Gezari et al. 2012). Middle: X-ray light curve obtained with Swift/XRT, NICER, and XMM-Newton. The spread in NICER measurements is real, tracing dramatic and fast variability (order of magnitude changes seen day-to-day). The horizontal dotted line marks the 2011 May archival flux level in the XMM-Newton 0.3-10 keV band (Gallo et al. 2013). Top: equivalent-width evolution of the broad components of the $\mathrm{H} \beta$ and $\mathrm{H} \alpha$ emission lines.

(The data used to create this figure are available.) 


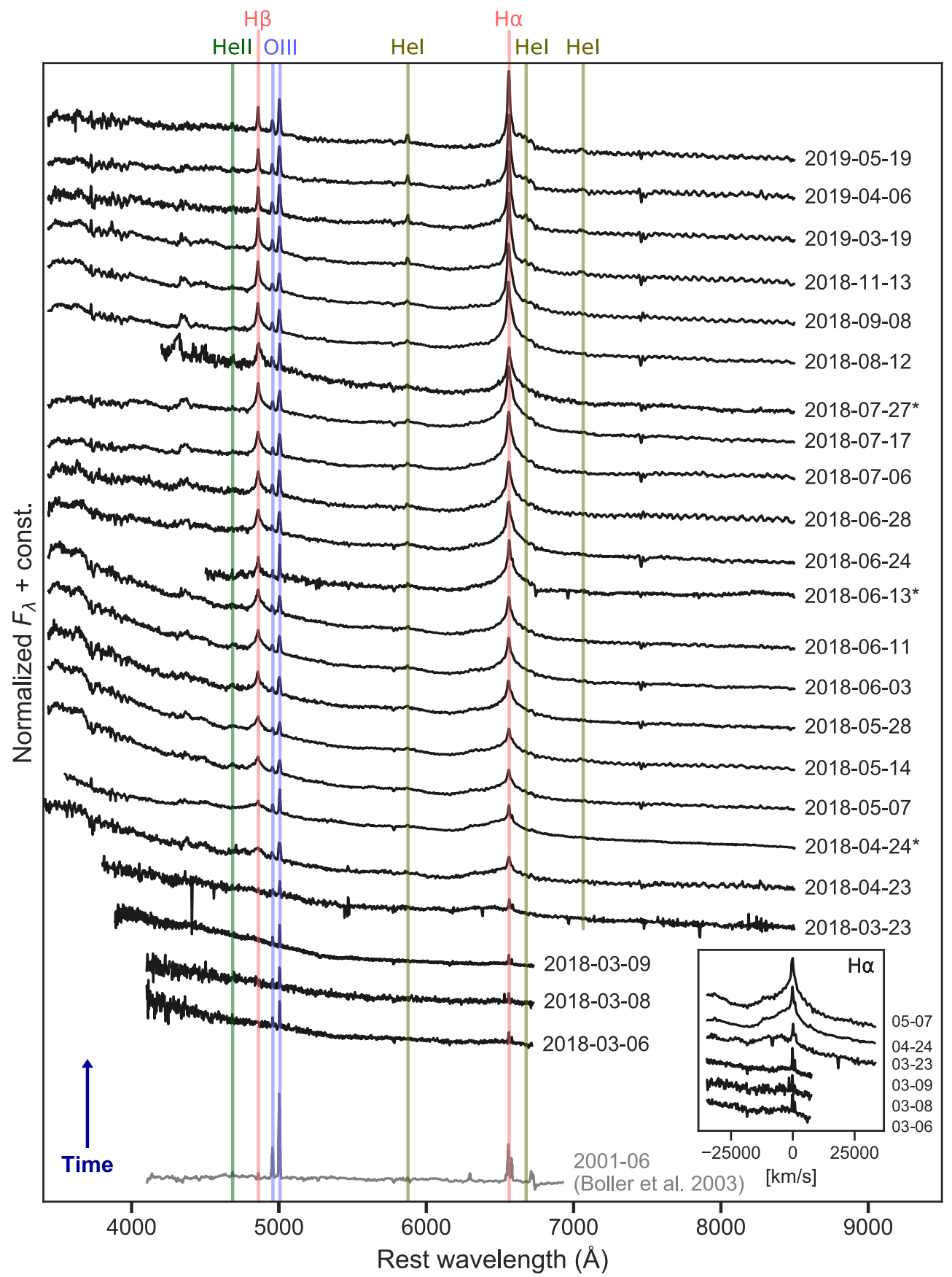

Figure 3. Optical spectra of 1ES 1927+654 showing the transition from a narrow-line (Type-2) AGN (bottom; from Boller et al. 2003) to a broadline (Type-1) AGN with an intermediate stage of a blue-continuum-dominated emission. The appearance of the broad lines is constrained to a timescale of several weeks (between the 2018 March 6 and April 23 spectra), although there is some evidence for a weak, broad, and blueshifted $\mathrm{H} \alpha$ line in our first spectra, as shown in the inset. All spectra are plotted without Milky Way extinction corrections. Dates marked with $*$ indicate spectra taken under different weather and/or instrument conditions compared to a standard star, thus affecting the validity of their continuum shape.

(The data used to create this figure are available.)

Telescope Imaging Spectrograph (STIS) instruments, respectively, on board the Hubble Space Telescope (HST; Program ID 15604, PI: C.L. MacLeod). Each spectrum was obtained through single-orbit exposures of 2866 and $2740 \mathrm{~s}$, respectively, on August 28, and reduced following standard HST procedures. The two flux-calibrated $H S T$ spectra are shown in Figure 4.

A $\log$ of all our spectral observations of 1ES $1927+654$ is detailed in Table 1 and all our spectra are available for download from the Weizmann Interactive Supernova Data Repository (WISeREP). ${ }^{25}$

We also obtained optical spectra of the four nearest (projected) neighboring sources of 1ES 1927+654 (sources within $\sim 3^{\prime \prime}-12^{\prime \prime}$, numbered 2, 3, 5, and 6 in Figure 9 of Boller et al. 2003), using the FLOYDS spectrograph on the Las

25 http://wiserep.weizmann.ac.il/ 


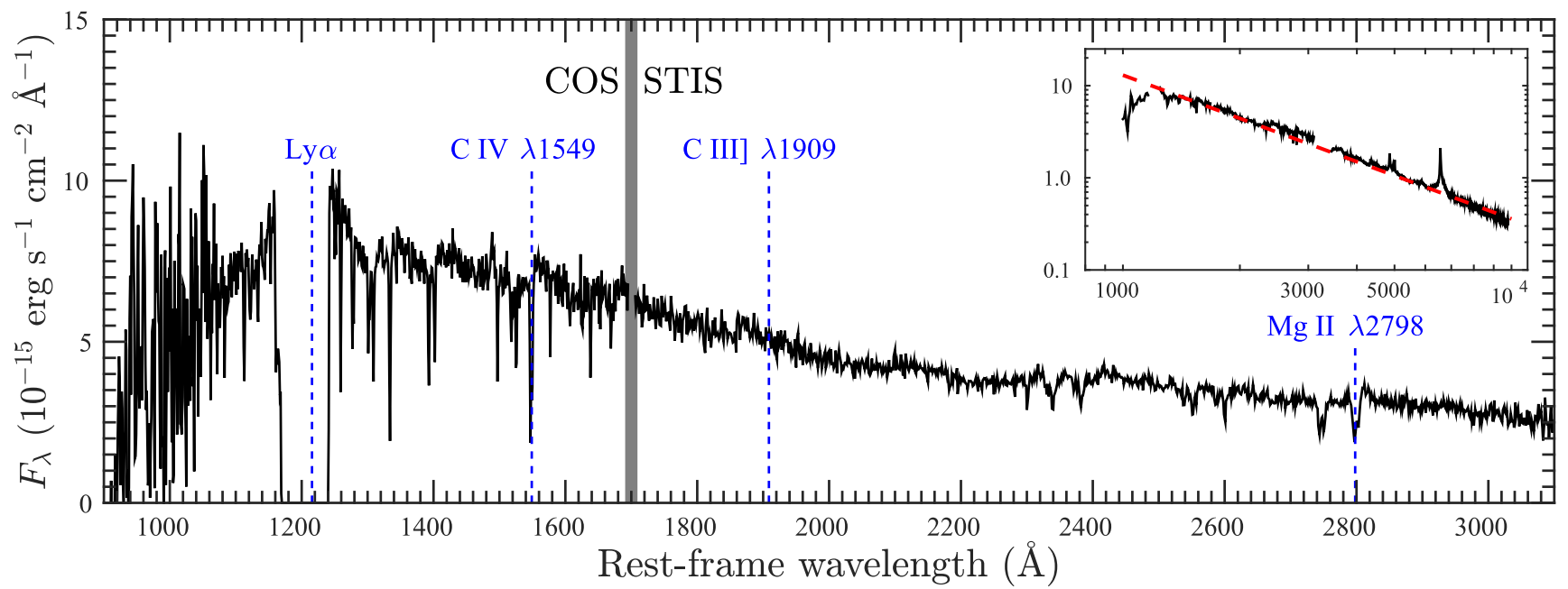

Figure 4. A combined HST UV spectrum of 1ES $1927+654$, obtained on 2018 August 28 with the COS (shortward of rest-frame wavelength of $\lambda_{\text {rest }}=1700 \AA$ ) and STIS (longward of $1700 \AA$ ) instruments. The vertical lines mark the expected locations of the C IV $\lambda 1549, \mathrm{C}$ III] $\lambda 1909$, and Mg II $\lambda 2798$ transitions, commonly seen as prominent broad $\left(>1000 \mathrm{~km} \mathrm{~s}^{-1}\right)$ emission lines in broadline AGNs. The absence of such broad lines is consistent with the presence of dust in the BLR. The inset compares the UV-optical spectral energy distribution (SED), which includes the HST spectra and the 2018 September 8 Las Cumbres spectrum (all median-binned to $7 \AA$ ), with a typical quasar power-law SED of the form $f_{\nu} \propto \nu^{-0.44}$ (Vanden Berk et al. 2001). This agreement indicates a mostly dust-free line of sight toward the continuum source.

Table 1

Log of Spectroscopic Observations

\begin{tabular}{|c|c|c|c|c|c|}
\hline Date $^{a}$ & $\begin{array}{c}\text { MJD Start } \\
\text { Time }^{\text {a }} \text { (days) }\end{array}$ & $\begin{array}{c}\Delta t_{\text {rf }}{ }^{\mathrm{b}} \\
\text { (days) }\end{array}$ & Telescope & Inst. & $\begin{array}{c}\text { Exp. } \\
\text { Time (s) }\end{array}$ \\
\hline 2018 Mar 6 & 58183.5 & 72 & MDM Hiltner $2.4 \mathrm{~m}$ & OSMOS & 600 \\
\hline 2018 Mar 8 & 58185.5 & 74 & MDM Hiltner $2.4 \mathrm{~m}$ & OSMOS & 1800 \\
\hline 2018 Mar 9 & 58186.5 & 75 & MDM Hiltner $2.4 \mathrm{~m}$ & OSMOS & 2700 \\
\hline $2018 \operatorname{Mar}_{13}{ }^{\mathrm{c}}$ & 58190.0 & 79 & Liverpool Telescope $2 \mathrm{~m}$ & SPRAT & 600 \\
\hline 2018 Mar $23^{*}$ & 58200.5 & 89 & APO $3.5 \mathrm{~m}$ & DIS & 300 \\
\hline 2018 Apr 23 & 58231.6 & 119 & Las Cumbres $2 \mathrm{~m}$ & FLOYDS & 1800 \\
\hline 2018 Apr 24 & 58232.5 & 120 & Lick Shane $3 \mathrm{~m}$ & Kast & $\sim 1500^{\mathrm{d}}$ \\
\hline 2018 May 7 & 58245.6 & 133 & Las Cumbres $2 \mathrm{~m}$ & FLOYDS & 1800 \\
\hline 2018 May 14 & 58252.5 & 140 & Las Cumbres $2 \mathrm{~m}$ & FLOYDS & 1800 \\
\hline 2018 May 28 & 58266.5 & 153 & Las Cumbres $2 \mathrm{~m}$ & FLOYDS & 1800 \\
\hline 2018 Jun 3 & 58272.6 & 159 & Las Cumbres $2 \mathrm{~m}$ & FLOYDS & 1800 \\
\hline 2018 Jun 11 & 58280.6 & 167 & Las Cumbres $2 \mathrm{~m}$ & FLOYDS & 1800 \\
\hline 2018 Jun $13^{*}$ & 58282.5 & 169 & APO $3.5 \mathrm{~m}$ & DIS & 600 \\
\hline 2018 Jun 24 & 58293.3 & 180 & Las Cumbres $2 \mathrm{~m}$ & FLOYDS & 1800 \\
\hline 2018 Jun 28 & 58297.5 & 184 & Las Cumbres $2 \mathrm{~m}$ & FLOYDS & 3600 \\
\hline 2018 Jul 6 & 58305.5 & 192 & Las Cumbres $2 \mathrm{~m}$ & FLOYDS & 3600 \\
\hline 2018 Jul 16 & 58315.5 & 201 & Keck $10 \mathrm{~m}$ & LRIS & 900 \\
\hline 2018 Jul 17 & 58316.5 & 202 & Las Cumbres $2 \mathrm{~m}$ & FLOYDS & 3600 \\
\hline 2018 Jul 27 & 58326.5 & 212 & Las Cumbres $2 \mathrm{~m}$ & FLOYDS & 3600 \\
\hline 2018 Aug 11 & 58341.0 & 227 & Palomar Hale $5 \mathrm{~m}$ & DBSP & 1200 \\
\hline 2018 Aug 12 & 58342.4 & 228 & Las Cumbres $2 \mathrm{~m}$ & FLOYDS & 3600 \\
\hline 2018 Sep 8 & 58369.3 & 254 & Las Cumbres $2 \mathrm{~m}$ & FLOYDS & 3600 \\
\hline 2018 Nov 13 & 58435.2 & 319 & Las Cumbres $2 \mathrm{~m}$ & FLOYDS & 3600 \\
\hline 2019 Mar 19 & 58561.5 & 443 & Las Cumbres $2 \mathrm{~m}$ & FLOYDS & 2700 \\
\hline 2019 Apr 6 & 58579.5 & 461 & Las Cumbres $2 \mathrm{~m}$ & FLOYDS & 2700 \\
\hline 2019 May 19 & 58622.6 & 503 & Las Cumbres $2 \mathrm{~m}$ & FLOYDS & 2700 \\
\hline 2018 Aug 28 & 58358.4 & 244 & $H S T 2.4 \mathrm{~m}$ & $\cos$ & 2866 \\
\hline 2018 Aug 28 & 58358.5 & 244 & $H S T 2.4 \mathrm{~m}$ & STIS & 2740 \\
\hline
\end{tabular}

Notes.

${ }^{\text {a }}$ At exposure start, UT. Dates marked with $*$ indicate spectra taken under different weather and/or instrument conditions compared to a standard star, thus affecting the validity of their continuum shape.

b Time since ATLAS first detection of the optical flare, in rounded rest-frame days.

${ }^{\mathrm{c}}$ A low-resolution spectrum, not shown in Figure 3.

${ }^{\mathrm{d}}$ The Shane/Kast exposure times were $3 \times 500 \mathrm{~s}$ for the red arm, and $1 \times 1560 \mathrm{~s}$ for the blue. 
Cumbres Observatory 2 m Faulkes Telescope North. We find that all sources are Milky Way stars, which are not expected to contribute to the significant activity detected toward 1ES 1927 $+654{ }^{26}$

\section{Analysis}

The NUV emission from 1ES 1927+654, as measured from our first Swift observations, has increased by a factor of about 40 compared with the 2011 May XMM-Newton measurement (Gallo et al. 2013). The X-ray Telescope (XRT) data (Figure 2), together with new data we obtained with XMMNewton and the Neutron star Interior Composition ExploreR (NICER; Gendreau et al. 2012), show more complex behavior compared to the optical and UV light curve, including significant variability on timescales of hours seen in the NICER rapid monitoring. These X-ray data are analyzed in detail in a companion paper (C. Ricci et al. 2019, in preparation).

The first post-flare optical spectra are dominated by blue continuum emission, as well as several narrow emission lines, including $\mathrm{H} \beta$, [O III] $\lambda 5007, \mathrm{H} \alpha$, and $[\mathrm{N}$ II] $\lambda 6584$. There is evidence for a weak, broad, and blueshifted $\mathrm{H} \alpha$ emission feature in these first post-flare spectra (see inset of Figure 3), however the limited spectral coverage does not allow for a robust measurement. The narrow-line emission is consistent with gas photoionized by an AGN-like continuum, according to emission-line ratio diagnostics (i.e., "BPT diagnostics;" see, e.g., Kewley et al. 2006, and references therein), and is therefore consistent with previous studies of 1ES 1927+654. However, the blue continuum was not present in archival data (Boller et al. 2003; Tran et al. 2011).

Strong, broad $\mathrm{H} \alpha$ and $\mathrm{H} \beta$ lines appeared on top of the blue continuum between 2018 March 6 and April 23 (Figure 3). These lines remain strong during our entire spectroscopic monitoring campaign, lasting at least 11 months after the optical flare detection (see top panel of Figure 2). To our knowledge this is the first time an AGN has been caught in the act of changing its type, with a blue continuum seen to appear before the broad lines, and the timescale for the broadline appearance constrained to within about a month (i.e., between the 2018 March 23 and April 23 spectra). The strength of the broad emission lines seems to evolve in a way that is generally similar to the UV/optical continuum emission (bottom panel of Figure 2), peaking with a delay of roughly 150 (rest frame) days after the continuum peak, and then (slowly) declining. The rise of the broad lines is, however, much slower than that of the continuum.

The FUV (HST/COS) spectrum shows evidence for broad Ly $\alpha$ line emission, although the core of the line is missing owing to the physical gap in the COS detectors. Most notably, the HST spectra do not show the prominent broad C IV $\lambda 1549$, C III] $\lambda 1909$, and Mg II $\lambda 2798$ emission lines, commonly seen in the spectra of "normal," persistent broadline AGNs (quasars). The combined UV (HST, 2018 August 28) and optical (Las Cumbres, 2018 September 8) post-flare spectrum of 1ES $1927+654$ is in excellent agreement with the typical spectral shape of such broadline AGNs (i.e., $f_{\nu} \sim \nu^{-0.5}$, Vanden Berk et al. 2001; see inset of Figure 4). Moreover, the UVoptical spectral energy distribution (SED) —as probed through the Swift/UVOT UVM2 $-V$ color measurements-shows

\footnotetext{
${ }^{26}$ None of these stars are detected in the Chandra data of Boller et al. (2003).
}

very little evolution during our monitoring. All this justifies some of the spectral analysis steps detailed below.

To measure the spectral features observed in our optical data, we decomposed the spectral regions surrounding the $\mathrm{H} \beta, \mathrm{Mg}$ II, and CIV lines, following the procedures presented by Trakhtenbrot \& Netzer (2012). The region surrounding the $\mathrm{H} \alpha$ line was decomposed using a procedure that follows the one presented by Mejía-Restrepo et al. (2016). The spectral models include a local (linear) continuum, a broadened iron emission template, single Gaussians for the narrow features of the $\mathrm{H} \beta$, [O III], $\mathrm{H} \alpha$, and [N II] lines, and two broad Gaussians for each of the broad emission lines. Uncertainties on key quantities were derived through a resampling procedure, fitting 100 realizations of each of the observed spectra assuming the observed noise, and eventually adopting the 16th and 84th quantiles as $1 \sigma$ equivalent errors on the quantities in question.

The top panel of Figure 2 shows the time evolution of the rest-frame equivalent widths (EW) of the broad $\mathrm{H} \beta$ and $\mathrm{H} \alpha$ lines. There is a clear rise in the broad $\mathrm{EW}(\mathrm{H} \alpha)$ between 2018 March 9 and 23 (i.e., about 70-90 days after the optical transient detection), completing an order-of-magnitude increase to $\mathrm{EW}(\mathrm{H} \alpha) \approx 110 \AA$ by April 23 (120 days after detection, in the rest frame). By this time the broad $\mathrm{H} \beta$ line can be robustly identified, with $\mathrm{EW}(\mathrm{H} \beta)=16 \pm 2 \AA$. Upon first robust appearance of a broad $\mathrm{H} \alpha$ line (in the March 23 spectrum), it is highly blueshifted, with $\Delta v \approx-5000 \mathrm{~km} \mathrm{~s}^{-1}$ compared to the systemic redshift (and to the narrow $\mathrm{H} \alpha$ component), and broad, with a full width at half-maximum intensity (FWHM) of $\operatorname{FWHM}(\mathrm{H} \alpha) \approx 18,000 \mathrm{~km} \mathrm{~s}^{-1}$. At later times the line emission remains roughly constant, with $\mathrm{EWs}$ in the range $\mathrm{EW}(\mathrm{H} \beta) \approx$ $15-27 \AA$ and $\mathrm{EW}(\mathrm{H} \alpha) \approx 125-180 \AA$. The $\mathrm{H} \alpha$ strength in 1ES $1927+654$ is rather typical of low-redshift broadline AGNs, with roughly half of such systems having $\operatorname{EW}(\mathrm{H} \alpha) \lesssim$ $200 \AA$ (see, e.g., the luminous AGN samples studied by Shen et al. 2011 and Koss et al. 2017, and also Stern \& Laor 2012a and $\mathrm{Oh}$ et al. 2015 for less-luminous systems). The $\mathrm{H} \beta$ emission in 1ES 1927+654, on the other hand, is rather weak, compared with the $\sim 95 \%$ of vigorously accreting broadline, low-redshift AGNs that have $\operatorname{EW}(\mathrm{H} \beta)>25 \AA$ (see also Trakhtenbrot \& Netzer 2012).

Throughout our spectroscopic monitoring, the broad $\mathrm{H} \alpha$ to $\mathrm{H} \beta$ flux ratio, which probes the Balmer decrement, was high: it reached $F(b \mathrm{H} \alpha) / F(b \mathrm{H} \beta) \gtrsim 8$ about 150 days after the transient detection (in the rest frame), and further increased up to $\gtrsim 15$, as the broad $\mathrm{H} \beta$ line flux was decreasing. These line ratios are much higher than what is typically seen in broadline AGNs (e.g., Dong et al. 2008), and are indicative of a highly dusty BLR. This can also account for the nondetection of UV line emission: our $H S T$ spectra resulted in upper limits of $\mathrm{EW}(\mathrm{C}$ IV $)<1 \AA$ and $\mathrm{EW}(\mathrm{Mg}$ II $)<5 \AA$, which are indeed much lower that what is usually found for broadline AGNs (e.g., Baskin \& Laor 2005; Sulentic et al. 2007; Richards et al. 2011; Shen et al. 2011; Tang et al. 2012). The steep Balmer decrement suggests extinction levels of at least $A_{\text {line }} \approx 6.5$ and 4.8 mag at the central wavelengths of the C IV and Mg II lines, respectively. ${ }^{27}$ If one would scale up our UV line EW upper limits based on these significant levels of extinction, then our data could be consistent with the distribution of broad UV emission line strength, as seen in normal broadline AGNs. Recalling that the continuum emission does not show any

\footnotetext{
${ }^{27}$ Assuming again the Cardelli et al. (1989) extinction law and $R_{V}=3.1$.
} 
evidence for significant dust extinction (inset of Figure 4; but see also Baron et al. 2016), we conclude that a high dust content within the BLR is consistent with all the UV-optical data in hand. This combination is quite rare in normal, persistent broadline AGNs (e.g., Baron et al. 2016). However, it could perhaps be expected if the broad emission lines originate from gas in which the dust in the parts closer to the central engine were exposed to a recent "flash" of sublimating (UV) continuum radiation. We discuss this point further in Section 4.

To derive rough estimates of the size of the newly detected broad-emission-line region, $R_{\mathrm{BLR}}$, we rely on $R_{\mathrm{BLR}}-L$ relations derived from reverberation mapping campaigns. As the broadline emission is essentially driven by the ionizing (UV) radiation from the central engine, we can use the highest Swift-measured NUV monochromatic luminosity ${ }^{28}$ of $\lambda L_{\lambda}(\mathrm{NUV}) \approx 1.2 \times$ $10^{44} \mathrm{erg} \mathrm{s}^{-1}$ (in the $U V M 2$ band, $\lambda_{\text {eff }}=2262 \AA$ ), and the $R_{\mathrm{BLR}}$ $-L_{1450}$ relation of Kaspi et al. (2005), to derive a BLR size of $R_{\mathrm{BLR}}(\mathrm{UV}) \approx 30$ lt-day. This estimate is likely a lower limit, as our earliest Swift observation took place after the optical peak (see Figure 2), and since the SED of the source is likely to further rise from the observed NUV band toward, and indeed beyond, the Lyman limit (see Shull et al. 2012, and references therein).

Using instead the optical monochromatic luminosity at rest frame $5100 \AA, \lambda L_{\lambda}\left(5100 \AA\right.$ ) (hereafter $\left.L_{5100}\right)$, measured from the first spectrum that robustly shows both broad Balmer emission lines (the 2018 April 23 spectrum), which is $L_{5100}=9.6 \times 10^{42} \mathrm{erg} \mathrm{s}^{-1}$, and the $R_{\mathrm{BLR}}-L_{5100}$ prescription of Bentz et al. (2013), we derive $R_{\mathrm{BLR}} \approx 10$ lt-day.

The BLR sizes we obtain through $R_{\mathrm{BLR}}-L$ relations, on the order of tens of light days, are consistent with the delay of 1-3 months between the UV/optical flux increase and the appearance of the broad lines, to within the uncertainties related with the $R_{\mathrm{BLR}}-L$ relations, which are mostly due to systematics. Indeed, the source-to-source scatter around the $R_{\mathrm{BLR}}-L$ relations is of order 0.2 dex (e.g., Bentz et al. 2013).

We further use the Keck/LRIS spectrum taken on 2018 July 16 to estimate key properties of the accreting SMBH powering $1 E S 1927+654$. This higher resolution spectrum probes the broad Balmer lines about 12 weeks after their appearance. The best-fit spectral model results in a broad $\mathrm{H} \beta$ line width of $\operatorname{FWHM}(\mathrm{H} \beta)=3100_{-80}^{+70} \mathrm{~km} \mathrm{~s}^{-1}$. The (narrow) [O III] $\lambda 5007$ line peak indicates a redshift of 0.01942 , which we adopt throughout. Combining the aforementioned $L_{5100}$-based BLR size estimate of 10 lt-day with the broad $\mathrm{H} \beta$ width, and a virial factor $f=1$, we obtain a virial ("single-epoch") BH mass estimate of $M_{\mathrm{BH}} \approx 1.9 \times 10^{7} M_{\odot}$. The uncertainties on such mass estimates are of order 0.3-0.5 dex-dominated by systematic uncertainties on the $R_{\mathrm{BLR}}-L_{5100}$ relation and on the virial factor $f$ (see Shen 2013 for a detailed discussion). Our $M_{\mathrm{BH}}$ estimate is in excellent agreement with the one reported by Tran et al. $\left(2011 ; 2.2 \times 10^{\top} M_{\odot}\right)$, which was based on far less robust methods (i.e., the narrow [O III] $\lambda 5007$ line).

Using the aforementioned 2018 April 23 measurement of $L_{5100}$ and a bolometric correction of $f_{\text {bol }}(5100 \AA)=9$ (e.g., Kaspi et al. 2000; Runnoe et al. 2012), we derive a bolometric luminosity of $L_{\mathrm{bol}}=8.6 \times 10^{43} \mathrm{erg} \mathrm{s}^{-1}$, close to the highest optical flux levels covered by our observations (about 120 restframe days after the transient discovery). Combining this with our

\footnotetext{
$\overline{28 \text { We assume a cosmological model with }} \Omega_{\Lambda}=0.7, \Omega_{\mathrm{M}}=0.3$, and $H_{0}=$ $70 \mathrm{~km} \mathrm{~s}^{-1} \mathrm{Mpc}^{-1}$
}

$M_{\mathrm{BH}}$ estimate, we derive an eddington ratio of $L / L_{\mathrm{Edd}} \equiv f_{\mathrm{bol}}$ $(5100 \AA) \times L_{5100} /\left(1.5 \times 10^{38} \times\left[M_{\mathrm{BH}} / M_{\odot}\right]\right) \approx 0.03$. Adopting the same $M_{\mathrm{BH}}$ estimate and bolometric correction to all our spectroscopic data (i.e., $L_{5100}$ measurements), we obtain eddington ratios in the range $L / L_{\text {Edd }} \approx 0.008-0.03$. If we instead use the highest measured NUV luminosity and a conservative NUV bolometric correction of $f_{\mathrm{bol}}(\mathrm{NUV})=2$ (or 4 ; see, e.g., Runnoe et al. 2012; Trakhtenbrot \& Netzer 2012; Netzer et al. 2016), we obtain $L_{\text {bol }} \approx 2.3 \times 10^{44} \mathrm{erg} \mathrm{s}^{-1}\left(\right.$ or $\left.4.6 \times 10^{44} \mathrm{erg} \mathrm{s}^{-1}\right)$, and eddington ratios of order $L / L_{\mathrm{Edd}} \approx 0.1$ (or 0.2 , respectively). All these estimates of $L_{\mathrm{bol}}$ and $L / L_{\mathrm{Edd}}$ are highly uncertain, as the (optical to x-ray) SED shape of 1ES 1927+654 during this intensified UV/optical emission episode, and thus the bolometric luminosity and corrections, are likely very different from what is normally seen and/or assumed for broadline AGNs. A detailed analysis of the full SED of 1ES $1927+654$ during the transient event presented here, as well as the implied $L_{\text {bol }}$ and $L / L_{\text {Edd }}$, and a comparison to previous studies, will be presented in the companion paper ( $\mathrm{R}$. Li et al. 2019, in preparation).

\section{Discussion and Conclusion}

Our data show that 1 ES $1927+654$ experienced a dramatic increase in continuum UV/optical emission, forming a blue, AGN-like continuum, which was then followed by the appearance of prominent broad Balmer emission lines. While both the (optical) continuum and line emission rose within about a month, the appearance of the broad Balmer lines lagged the continuum rise by 1-3 months. The uncertainty in the timelag determination is due to the uncertainty in the exact time the UV flux peaked, and the exact time the broad lines emerged. Still, to our knowledge, this is the first observation of a CLAGN where the lag between the change in continuum and in broadline emission has been temporally resolved. Broad UV lines, some of which probe higher levels of ionization (e.g., C IV $\lambda 1549)$, remain undetected.

The delay between the continuum and broadline emission, if taken as the light travel time to the BLR, allows us to estimate a BLR radius $\left(R_{\mathrm{BLR}}\right)$ of roughly $1-3$ light-months. Moreover, our spectroscopic time series (Figure 3) shows that the highestvelocity BLR gas was observed to respond first, followed later by a steady increase in the lower-velocity, core line emission.

These dramatic changes seen in 1ES 1927+654 are unlikely to be driven by a change in the level of line-of-sight obscuration (i.e., a dusty cloud moving out of the line of sight), as in such a case one would expect no delay between the appearance of the two emission components. Indeed, our rich collection of X-ray data (C. Ricci et al. 2019, in preparation) offers no evidence for a coherent and persistent X-ray spectral change that could straightforwardly be linked to a change in line-of-sight obscuration. We also recall that 1ES $1927+654$ was identified as one of the few "true type-2" $\mathrm{AGN}^{29}$ - systems which show neither broadline emission nor significant line-ofsight obscuration (which might have accounted for the lack of broad lines). Such systems are thought to have either a low content of broadline emitting circumnuclear gas (i.e., no BLR gas) and/or insufficient levels of ionizing continuum emission (see Stern \& Laor 2012a, 2012b and references therein).

All this suggests that the changes seen in 1ES 1927+654 are most likely driven by a sudden change in the accretion flow onto the $\mathrm{SMBH}$, resulting in increased $\mathrm{UV} /$ optical continuum

\footnotetext{
${ }^{29}$ It most obviously no longer fits into this class.
} 
emission which traveled to the BLR gas, where it was reprocessed and gave rise to broadline emission.

Here we briefly consider three possibilities for the nearly cotemporaneous appearance of the blue continuum and broadline emission. We then turn to briefly discuss some of the mechanisms that could be driving the entire (enhanced emission) event, including the tidal disruption of a star.

First, a fresh supply of (cold) gas may have reached the close vicinity of the SMBH. Some of the gas may have ended up being accreted onto the SMBH, thus illuminating circumnuclear gas on larger scales, some of which has properties consistent with that of the BLR seen in "normal" broadline AGNs. Given the $L_{\text {bol }}$ estimates available for 1ES $1927+654$, the amount of gas that went through the AGN-like accretion flow during our monitoring is of order $\lesssim 0.01 M_{\odot}$ (assuming a standard radiative efficiency of $\eta=0.1$ ), and the total mass in the broad emission line may be of order $\sim 0.1 M_{\odot}$ (e.g., Netzer 2013).

However, the X-ray and narrow line emission previously seen in 1ES $1927+654$ indicate that the SMBH has been vigorously accreting for over a decade (and perhaps through $\sim 10^{3-4} \mathrm{yr}$ ). Moreover, the month-long rise of the continuum and line emission is far faster than what is expected from a global rise in the accretion rate through an AGN-like disk, and/ or from heating/cooling fronts traveling within such a disk 1ES 1927+654 (see discussion in, e.g., LaMassa et al. 2015; Lawrence 2018; Ross et al. 2018; Stern et al. 2018).

Second, a drastic change in the accretion flow and in the related UV/optical continuum emission may have initiated broadline emission in a preexisting reservoir of BLR-like gas. This can be the result of enhanced accretion through a preexisting accretion disk, although the observed timescales suggest that other, localized disturbances are much more likely (see the discussion in, e.g., Ross et al. 2018; Stern et al. 2018 and references therein). One intriguing possibility is that a UV "flash" sublimated the dust in the inner regions of a dusty obscuring structure (i.e., a dusty "torus"), making this innertorus region appear as a de-facto newly-formed BLR (see the review by Netzer 2015, and references therein). Given the previous "true Type-2" classification of 1ES 1927+654, it is possible that the system is observed at a relatively small inclination angle (i.e., relatively "face on"), and that the historically low UV emission allowed the torus to extend inwards. Indeed, the BLR gas may still have a significant dust content, which would account for the steep Balmer decrement and the nondetected broad UV emission lines. We recall again that the physical conditions and radiation transfer within the BLR gas in 1ES $1927+654$ may not be necessarily similar to those in normal broadline AGNs.

Finally, several models suggest that increased $\mathrm{SMBH}$ accretion may trigger disk instabilities (Nicastro et al. 2003) or indeed launch a disk-wind (e.g., Elitzur \& Ho 2009; Elitzur $\&$ Netzer 2016), either of which would then be exposed to the incident ionizing radiation (from the inner disk) and thus be seen in broadline emission. Some recent studies suggest observational evidence for a link between CL-AGN and such disk-winds (e.g., Giustini et al. 2017; MacLeod et al. 2019). However, the relevance of this disk-wind scenario to the drastic changes seen in 1ES $1927+654$ is unclear, given (1) the absence of high-ionization, UV broad emission lines (Figure 4), which are expected to form in the inner region of a disk-wind (e.g., Richards et al. 2011, and references therein); (2) the delay between the continuum and broadline emission appearance is much longer than what is expected for a disk-wind; and (3) the lack of clear evidence for outflows in our UV and optical spectroscopy. Moreover, the Nicastro et al. (2003) scenario is rather unlikely, as it is expected to occur around a threshold Eddington rate of $L / L_{\mathrm{Edd}} \approx 10^{-3}$-which is lower than the pre-flare state of 1ES 1927+654 (see Elitzur \& Netzer 2016).

The initial cause of the dramatic changes seen here remains to be determined. As noted above, the observed timescales are inconsistent with large-scale changes to an AGN-like accretion flow (i.e., a global, sudden change in $\dot{M}$ ), or to line-of-sight obscuration (i.e., $N_{\mathrm{H}}$ ).

Instead, the optical rise time, the peak luminosity, and the following decline are reminiscent of tidal disruption events (TDEs), as illustrated by the comparison to the TDE PS1-10jh (Gezari et al. 2012) and to a generic $t^{-5 / 3}$ power law (e.g., Rees 1988; Phinney 1989) shown in the bottom panel of Figure 2. The mass of the SMBH powering 1ES $1927+654$, of $M_{\mathrm{BH}} \approx 2 \times 10^{7} M_{\odot}$, is consistent with what is observed for SMBHs around which TDEs have been seen, but on the high end of the observed distribution (e.g., Wevers et al. 2017; van Velzen 2018; Wevers et al. 2019). Associating the changes in 1ES $1927+654$ with a TDE would be in line with the study of Merloni et al. (2015), which argued that another CL-AGN, where the blue continuum and broadline emission have disappeared within less than a decade (LaMassa et al. 2015), was driven by a (fading) TDE.

We note, however, that the freedom in setting the disruption time $\left(t_{\mathrm{d}}\right)$ - here set to the ATLAS (pre-discovery) detection of the optical transient on 2017 December 23-allows to fit such powerlaw behavior to a vast range of observed light curves. Moreover, the lack of extremely broad and strong He II $\lambda 4686$ line emission $\left(\mathrm{FWHM}[\mathrm{He}\right.$ II $\left.]>10,000 \mathrm{~km} \mathrm{~s}^{-1} ; F[\mathrm{He} \mathrm{II}] \gg F[\mathrm{H} \beta]\right)$, which is observed in TDEs during the first few months after their detection, as well as the spectral evolution of the continuum and Balmer lines, and the peculiar X-ray light curve, are all different from those seen in tidal disruptions occurring in inactive galaxies (see, e.g., Gezari et al. 2012; Arcavi et al. 2014; Holoien et al. 2014; Komossa 2015; Brown et al. 2017; Hung et al. 2017). While the nuclear transient PS16dtm, a recently claimed TDE in a narrow-line Seyfert 1 AGN (Blanchard et al. 2017), showed a drop in X-rays following the UV/optical transient that could be similar to what is seen in 1ES $1927+654$, there is no evidence that the X-ray emission has recovered as it did in our case.

A TDE in an AGN could indeed look very different from a simple combination of AGN- and TDE-like observables, given the interaction between the TDE and the preexisting accretion disk (see the recent study by Chan et al. 2019). Detailed modeling of such events is required for comparison with our observations.

Real-time identification and follow-up observations of events such as AT 2018zf/ASASSN-18el offer a spectacular opportunity to resolve the mystery of CL-AGN, and to improve our understanding of accretion and BLR physics. Fulfilling this potential would require high-cadence optical spectroscopy and complementary multiwavelength data, for a proper sample of such events. With the increasing number, cadence, and sky coverage of imaging time-domain surveys (e.g., ASAS-SN, ATLAS, ZTF, LSST) and responsive spectroscopic follow-up programs (e.g., using the Las Cumbres observatory network, ePESSTO, SDSS-V), such events will increasingly be caught in the act. 
We are grateful to the anonymous referee for helping us improve this manuscript. We thank Gwen Eadie, Bryce Bolin, and Dino Bektešević at UW for assistance with obtaining the APO spectra. We also thank Hagai Netzer, Dalya Baron, and Robert Antonucci for their insightful and constructive comments. R.L. was supported by the National Key R\&D Program of China (2016YFA0400702) and the National Science Foundation of China (11721303). I.A. acknowledges support from the Israel Science Foundation (grant No. 2108/18). Support for J.L.P. is provided in part by FONDECYT through the grant 1191038 and by the Ministry of Economy, Development, and Tourisms Millennium Science Initiative through grant IC120009, awarded to The Millennium Institute of Astrophysics, MAS. Support for A.V.F.'s research group has been provided by the TABASGO Foundation, the Christopher R. Redlich Fund, and the Miller Institute for Basic Research in Science (U.C. Berkeley).

Based on observations with the NASA/ESA/CSA Hubble Space Telescope obtained [from the Data Archive] at the Space Telescope Science Institute, which is operated by the Association of Universities for Research in Astronomy, Incorporated; under NASA contract NAS5-26555. Support for Cycle 25 Program GO-15604 was provided through a grant from the STScI under NASA contract NAS5-26555.

This work made use of the MATLAB package for astronomy and astrophysics (Ofek 2014) and Astropy, ${ }^{30}$ a communitydeveloped core Python package for Astronomy (The Astropy Collaboration et al. 2013, 2018). This research also made use of the NASA/IPAC Extragalactic Database (NED), which is operated by the Jet Propulsion Laboratory, California Institute of Technology, under contract with the National Aeronautics and Space Administration.

This work made use of data from Las Cumbres Observatory; the All Sky Automated Survey for Supernovae (ASAS-SN); the Asteroid Terrestrial-impact Last Alert System (ATLAS) project; the Liverpool Telescope; the Lick Observatory; and the W. M. Keck Observatory.

ATLAS is primarily funded to search for near earth asteroids (NEOs) through NASA grants NN12AR55G, 80NSSC18K0284, and 80NSSC18K1575; byproducts of the NEO search include images and catalogs from the survey area. The ATLAS science products have been made possible through the contributions of the University of Hawaii Institute for Astronomy, the Queen's University Belfast, the Space Telescope Science Institute, and the South African Astronomical Observatory. ASAS-SN is supported by the Gordon and Betty Moore Foundation through grant GBMF5490 to the Ohio State University and NSF grant AST-1515927. Development of ASAS-SN has been supported by NSF grant AST-0908816, the Mt. Cuba Astronomical Foundation, the Center for Cosmology and AstroParticle Physics at the Ohio State University (CCAPP), the Chinese Academy of Sciences South America Center for Astronomy (CASSACA), the Villum Foundation, and George Skestos. We thank the Las Cumbres Observatory and its staff for its continuing support of the ASAS-SN project. The Liverpool Telescope is operated on the island of La Palma by Liverpool John Moores University in the Spanish Observatorio del Roque de los Muchachos of the Instituto de Astrofisica de Canarias, with financial support from the UK Science and Technology Facilities Council. Research at Lick

\footnotetext{
30 http://www.astropy.org
}

Observatory is partially supported by a generous gift from Google. The W. M. Keck Observatory is operated as a scientific partnership among the California Institute of Technology, the University of California, and NASA; the observatory was made possible by the generous financial support of the W. M. Keck Foundation.

We finally thank NASA HEASARC for making the Swift and NICER data available, and ESA for providing the XMMNewton data.

Facilities: HST (STIS), HST (COS), Swift (XRT and UVOT), NICER, XMM, ATLAS, ASAS-SN, MDM Observatory (OSMOS), Liverpool Telescope (SPRAT), Lick Observatory (Shane/Kast), Las Cumbres Observatory (FLOYDS), Keck (LRIS).

Software: astropy (The Astropy Collaboration et al. 2013, 2018), SExtractor (Bertin \& Arnouts 1996), MAAT (Ofek 2014).

\section{ORCID iDs}

Benny Trakhtenbrot (ib https://orcid.org/0000-00023683-7297

Iair Arcavi (ib https://orcid.org/0000-0001-7090-4898 Claudio Ricci (i) https://orcid.org/0000-0001-5231-2645

Melissa L. Graham (iD https://orcid.org/0000-0002-9154-3136

Daniel Stern (iD https://orcid.org/0000-0003-2686-9241

Griffin Hosseinzadeh (iD https://orcid.org/0000-00020832-2974

D. Andrew Howell (1) https://orcid.org/0000-0003-4253-656X Stephen J. Smartt (iD https://orcid.org/0000-0002-8229-1731 Jose L. Prieto (iD https://orcid.org/0000-0003-0943-0026 Benjamin J. Shappee (10 https://orcid.org/0000-00034631-1149

Thomas W.-S. Holoien (10 https://orcid.org/0000-00019206-3460

David Bersier (iD https://orcid.org/0000-0001-7485-3020

Alexei V. Filippenko (i) https://orcid.org/0000-00033460-0103

WeiKang Zheng (DiD https://orcid.org/0000-0002-2636-6508

\section{References}

Alard, C. 2000, A\&AS, 144, 363

Alard, C., \& Lupton, R. H. 1998, ApJ, 503, 325

Antonucci, R. 1993, ARA\&A, 31, 473

Antonucci, R. 2018, NatAs, 2, 504

Arcavi, I., Gal-Yam, A., Sullivan, M., et al. 2014, ApJ, 793, 38

Baron, D., Stern, J., Poznanski, D., \& Netzer, H. 2016, ApJ, 832, 8

Baskin, A., \& Laor, A. 2005, MNRAS, 356, 1029

Bentz, M. C., Denney, K. D., Grier, C. J., et al. 2013, ApJ, 767, 149

Bertin, E., \& Arnouts, S. 1996, A\&AS, 117, 393

Bianchi, S., Antonucci, R., Capetti, A., et al. 2019, MNRAS, 488, L1 Blanchard, P. K., Nicholl, M., Berger, E., et al. 2017, ApJ, 843, 106 Boller, T., Voges, W., Dennefeld, M., et al. 2003, A\&A, 397, 557 Brown, J. S., Holoien, T. W.-S., Auchettl, K., et al. 2017, MNRAS, 466, 4904 Brown, T. M., Baliber, N., Bianco, F. B., et al. 2013, PASP, 125, 1031 Cardelli, J. A., Clayton, G. C., \& Mathis, J. S. 1989, ApJ, 345, 245 Chan, C.-H., Piran, T., Krolik, J. H., \& Saban, D. 2019, arXiv:1904.12261 Denney, K. D., De Rosa, G., Croxall, K., et al. 2014, ApJ, 796, 134 Dong, X.-B., Wang, T., Wang, J.-G., et al. 2008, MNRAS, 383, 581 Elitzur, M., \& Ho, L. C. 2009, ApJL, 701, L91

Elitzur, M., \& Netzer, H. 2016, MNRAS, 459, 585

Filippenko, A. V. 1982, PASP, 94, 715

Gallo, L. C., MacMackin, C., Vasudevan, R. V., et al. 2013, MNRAS, 433, 421

Gendreau, K. C., Arzoumanian, Z., \& Okajima, T. 2012, Proc. SPIE, 8443, 844313

Gezari, S., Chornock, R., Rest, A., et al. 2012, Natur, 485, 217

Giustini, M., Costantini, E., De Marco, B., et al. 2017, A\&A, 597, A66 
Henden, A. A., Templeton, M., Terrell, D., et al. 2016, yCat, 2336, 0 Holoien, T. W., Prieto, J. L., Bersier, D., et al. 2014, MNRAS, 445, 3263

Hung, T., Gezari, S., Blagorodnova, N., et al. 2017, ApJ, 842, 29

Kaspi, S., Maoz, D., Netzer, H., et al. 2005, ApJ, 629, 61

Kaspi, S., Smith, P. S., Netzer, H., et al. 2000, ApJ, 533, 631

Kewley, L. J., Groves, B., Kauffmann, G., \& Heckman, T. M. 2006, MNRAS, 372,961

Komossa, S. 2015, JHEAp, 7, 148

Koss, M. J., Trakhtenbrot, B., Ricci, C., et al. 2017, ApJ, 850, 74

LaMassa, S. M., Cales, S., Moran, E. C., et al. 2015, ApJ, 800, 144

Lawrence, A. 2018, NatAs, 2, 102

MacLeod, C. L., Green, P. J., Anderson, S. F., et al. 2019, ApJ, 874, 8

MacLeod, C. L., Ross, N. P., Lawrence, A., et al. 2016, MNRAS, 457, 389

Martini, P., Stoll, R., Derwent, M. A., et al. 2011, PASP, 123, 187

Matt, G., Guainazzi, M., \& Maiolino, R. 2003, MNRAS, 342, 422

McElroy, R. E., Husemann, B., Croom, S. M., et al. 2016, A\&A, 593, L8

Mejía-Restrepo, J. E., Trakhtenbrot, B., Lira, P., Netzer, H., \& Capellupo, D. M. 2016, MNRAS, 460, 187

Merloni, A., Dwelly, T., Salvato, M., et al. 2015, MNRAS, 452, 69

Miller, J. S., \& Stone, R. P. S. 1993, Lick Obs. Tech. Rep. 66, (Santa Cruz, CA: Lick Obs.)

Netzer, H. 2013, The Physics and Evolution of Active Galactic Nuclei (Cambridge: Cambridge Univ. Press)

Netzer, H. 2015, ARA\&A, 53, 365

Netzer, H., Lani, C., Nordon, R., et al. 2016, ApJ, 819, 123

Nicastro, F., Martocchia, A., \& Matt, G. 2003, ApJL, 589, L13

Nicholls, B., Brimacombe, J., Kiyota, S., et al. 2018, ATel, 11391

Ofek, E. O. 2014, MATLAB package for astronomy and astrophysics, Astrophysics Source Code Library, ascl:1407.005

Oh, K., Yi, S. K., Schawinski, K., et al. 2015, ApJS, 219, 1

Oke, J. B., Cohen, J. G., Carr, M., et al. 1995, PASP, 107, 375

Oke, J. B., \& Gunn, J. E. 1982, PASP, 94, 586

Oke, J. B., \& Gunn, J. E. 1983, ApJ, 266, 713

Phinney, E. S. 1989, in Proc. IAU Symp. 136, The Center of the Galaxy, ed. M. Morris (Dordrecht: Springer), 543

Piascik, A. S., Steele, I. A., Bates, S. D., et al. 2014, Proc. SPIE, 9147, 91478H
Piconcelli, E., Fiore, F., Nicastro, F., et al. 2007, A\&A, 473, 85

Rees, M. J. 1988, Natur, 333, 523

Ricci, C., Bauer, F. E., Arevalo, P., et al. 2016, ApJ, 820, 5

Richards, G. T., Kruczek, N. E., Gallagher, S. C., et al. 2011, AJ, 141, 167

Ross, N. P., Ford, K. E. S., Graham, M., et al. 2018, MNRAS, 480, 4468

Runnoe, J. C., Brotherton, M. S., \& Shang, Z. 2012, MNRAS, 422, 478

Runnoe, J. C., Cales, S., Ruan, J. J., et al. 2016, MNRAS, 455, 1691

Schlafly, E. F., \& Finkbeiner, D. P. 2011, ApJ, 737, 103

Shappee, B. J., Prieto, J. L., Grupe, D., et al. 2014, ApJ, 788, 48

Shen, Y. 2013, BASI, 41, 61

Shen, Y., Richards, G. T., Strauss, M. a., et al. 2011, ApJS, 194, 45

Shull, J. M., Stevans, M., \& Danforth, C. W. 2012, ApJ, 752, 162

Stanek, K. Z. 2018, TNSTR, 287

Stern, D., McKernan, B., Graham, M. J., et al. 2018, ApJ, 864, 27

Stern, J., \& Laor, A. 2012a, MNRAS, 423, 600

Stern, J., \& Laor, A. 2012b, MNRAS, 426, 2703

Sulentic, J. W., Bachev, R., Marziani, P., Negrete, C. A., \& Dultzin-Hacyan, D. 2007, ApJ, 666, 757

Tang, B., Shang, Z., Gu, Q., Brotherton, M. S., \& Runnoe, J. C. 2012, ApJS, 201, 38

The Astropy Collaboration, Price-Whelan, A. M., Sipőcz, B. M., et al. 2018, AJ, 156, 123

The Astropy Collaboration, Robitaille, T. P., Tollerud, E. J., et al. 2013, A\&A, 558, A33

Tonry, J. L., Denneau, L., Heinze, A. N., et al. 2018, PASP, 130, 064505

Trakhtenbrot, B., \& Netzer, H. 2012, MNRAS, 427, 3081

Tran, H. D., Lyke, J. E., \& Mader, J. A. 2011, ApJL, 726, L21

Urry, C. M., \& Padovani, P. 1995, PASP, 107, 803

van Velzen, S. 2018, ApJ, 852, 72

Vanden Berk, D. E., Richards, G. T., Bauer, A. E., et al. 2001, AJ, 122, 549

Wang, J., Xu, D. W., \& Wei, J. Y. 2018, ApJ, 858, 49

Wevers, T., Stone, N. C., van Velzen, S., et al. 2019, MNRAS, 487, 4136

Wevers, T., van Velzen, S., Jonker, P. G., et al. 2017, MNRAS, 471, 1694

Yang, Q., Wu, X.-B., Fan, X., et al. 2018, ApJ, 862, 109 